\title{
Valoración económica del servicio limnológico de la laguna Magdalena-Atillo, Riobamba-Ecuador
}

\section{Economic valuation of the limnetic service of Magdalena-Atillo lake - Riobamba Ecuador}

\author{
Mónica Susana Delgado Yánez¹, Miguel Martínez-Fresneda Mestre², Antonio Villaruel ${ }^{3}$
}

\section{Resumen}

El presente trabajo es una propuesta de sostenimiento socioeconómico del recurso hídrico de la laguna de Magdalena en el complejo lacustre Atillo del Parque Nacional Sangay en la provincia de Chimborazo, mediante el método indirecto de costos de viaje.

En este estudio se establecen indicadores espaciales de situación hídrica y con técnicas GIS se crean unidades homogéneas, con las cuales se encuentra la distancia óptima para aplicar la metodología de círculos concéntricos en el uso de la herramienta econométrica de costos de viaje.

De acuerdo al análisis de las imágenes, se puede concluir que el lago se encuentra en retroceso; sin embargo, mantiene el límite limnimétrico todavía estable. Por ello, se propone limitar las visitas a un número inferior a 300 mensuales y se propone costo oportunidad para el sostenimiento socioeconómico, a partir de las unidades homogéneas, donde se considera el precio, mediante la estimación de precio del mercado, siendo su resultado igual al costo del viaje + Costo del tiempo de viaje, tomando como base el tiempo de permanencia promedio que no supera un día, para el sostenimiento del ecosistema.

\section{Palabras clave}

Economía medioambiental, desarrollo sostenible, hidrología, Conservación ambiental, laguna, recursos hídricos,

Clasificación JEL: Q01 Desarrollo sostenible.

\begin{abstract}
The present paper is a proposal of socio-economic sustainability of the water resource of the Magdalena lagoon in the Atillo lake complex of the Sangay National Park in the province of Chimborazo, by means of the indirect method of travel costs.

In this study, spatial indicators of hydric situation are established and homogeneous units are established with GIS techniques, in order to find the optimal distance to apply the concentric circle methodology in the use of the econometric tool of travel costs.

According to the image analysis, it can be concluded that the lake is in retreat; however, it still has limnimetric limits that are still stable. Because of this, it is proposed to limit the number of visits to the area to less than 300 per month and cost opportunity is proposed, for socio-economic sustainability, from the homogeneous units, where the price is considered by estimating the market price, the result being equal to the cost of the trip + Cost of travel time, based on the average stay time that does not exceed one day for the support of the ecosystem
\end{abstract}

\section{Keywords}

Environmental economics, Sustainable development, Hydrology, Environmental conservation, Lagoons, Water resources.

\footnotetext{
$1 \quad$ Universidad Internacional SEK, Quito-Ecuador (monica.delgado@uisek.edu.ec).

2 Universidad Internacional SEK, Quito-Ecuador (mestre.martinezQuisek.edu.ec).

3 Economista independiente.
} 


\section{Introducción}

El complejo lacustre Atillo, dentro del Parque Nacional Sangay, se encuentra a una altura máxima de 3648 m.s.n.m., entre las lomas loma Punta, loma Mesa Rushca, laguna Cuyug, loma Filo Cuyug Hanua y Atillo Grande, y corresponde a un espacio considerado pantanoso, siendo la laguna Magdalena o Colay nuestro objeto de estudio, esta se encuentra localizada en la provincia de Chimborazo, en la ciudad de Guamote, parroquia de Cebadas en la comunidad San José de Atillo, el acceso a la laguna se lo realiza por la carretera Guamote-Macas, a una altitud de 3485 m.s.n.m. (Calles, 2013, p. 95-97).

Se conoce poco sobre los procesos hidrológicos en el área de estudio, debido en general a la falta de resultados cuantificables (Camacho, 2008), se tienen, sin embargo, estudios aislados de cuantificación de parámetros hidro-meteorológicos (EMAPAR, 2012), y el valor económico en la zona es clave para el fortalecimiento socioeconómico de la región (MAE, 2013), ya que a partir de la valoración económica de los bienes y servicios ambientales en el ámbito hidrológico se puede llegar a establecer propuestas de conservación no solo de la laguna, sino de la microcuenca (Álvarez, Fernández y Rua, 2009, p. 15, 18-23).

Es fundamental entender cuáles son los métodos directos e indirectos de valoración económica ambiental (Moreno, Joven y Escobar, 2001, p. 2-13), y la correcta interpretación de sus resultados (Tomasini, 2013, p. 2-10), pues estos coadyuvan a plantear mecanismos de conservación integral (Álvarez et al., 2009, p.15, 18-23), los que seguidos de un análisis técnico y socioeconómico contribuyen a la sustentabilidad ambiental.

Los procesos de decisión para la conservación, generalmente, están muy ligados a la viabilidad económica de uso o servicio (Mirasgedis y Diakoulaki, 1997, p. 54), encamina a ideas constructivas que aseguran la continuidad (World Bank, 1992, p. 13), en tanto que el análisis económico (Checkland y Scholes, 1990, p. 6) ayuda con procesos de decisión (Moreno et al., 2001, p. 2-13), integrando conceptos ambientales (Mirasgedis y Diakoulaki, 1997, p. 54).

Para este trabajo el concepto de sustentabilidad se ha incorporado a los conceptos de valoración económica (Tomasini, 2013), tomando en cuenta que la calidad ambiental es parte del desarrollo social y sostenible del medio geográfico de estudio, y puede ser un punto de partida para el desarrollo de políticas (World Bank, 1992, p.13).

La conservación de lagunas inicia con estrategias de conservación de vida silvestre (Lumbreras, 2002), siendo el sistema de mecanismos de pago por servicios ambientales (PSA), el más aceptado en la conservación de recursos hídricos de una región (Camacho, 2008), sobre todo, por ser un mecanismo de conservación que ha dado en cierta medida resultados tangibles.

Se tienen experiencias positivas en cuanto a la conservación de páramos y ecosistemas dependientes de ellos (Molina, Serrano y Váscones, 2009), basadas en la valoración económica de la zona para establecer valores de pago por servicios ambientales, como el caso de los bosques templados lluviosos de la región Sierra Ecuador (Oyarzún, Nahuelhual, y Núñez, 2005).

El objetivo fue establecer una propuesta de sostenimiento socioeconómico del recurso hídrico de la laguna de Magdalena en el complejo lacustre Atillo del Parque Nacional Sangay en la provincia de Chimborazo, mediante el método indirecto de costos de viaje

\section{Metodología}

Mediante trabajo de campo, por causa de la dificultad de acceso se realiza en seis campañas de ocho horas cada una para obtener la georeferenciación de puntos en el límite de la laguna, 
y se establece el volumen mediante una batimetría de campo ya que el agua subterránea, así como la laguna es considerada como reserva de agua para poblaciones de Riobamba y Alausí e inclusive de sus alrededores.

Se utiliza la fórmula de Thorntwaite modificada por la FAO, ya que basa su teoría en la temperatura media y la altitud (ORSTOM-Francia, 1982 y Ravelo-FAO, 2000), tomando en cuenta la precipitación, que se puede ver dos estaciones definidas: una por un período de abundantes lluvias comprendido entre enero y abril, y el segundo período presenta menos precipitación entre los meses de mayo a diciembre, y un pico lluvioso menor en los meses de septiembreoctubre tiempo en el que se realizó la mayor parte de trabajo de campo.

Los valores de precipitación son puntuales, se los realiza con la serie de promedios anuales (serie 1985-2009), se calcula su distribución espacial en la zona en estudio; motivo por el cual se utiliza trazos de isoyetas, con un proceso de geoestadística con separación de $100 \mathrm{~mm}$ con valores que varía de $500 \mathrm{~mm}$ a $1100 \mathrm{~mm}$.

En lo referente a la temperatura, este parámetro es el de mayor importancia para el análisis económico, debido a que se su variación afecta de manera directa su riqueza vegetal, forestal y biológica; la estación climática considerada es Pachamama Tixán M135, de donde se obtienen temperaturas medias mensuales y anuales en el mismo período histórico, se considera para la precipitación y que varía desde los $7.6^{\circ} \mathrm{C}$ a $8.2^{\circ} \mathrm{C}$, con un promedio anual $8{ }^{\circ} \mathrm{C}$.

La investigación se lleva a cabo con el método econométrico de costos de viaje, realizada en dos etapas: 1. Recopilación de información para generar indicadores espaciales, y 2. Análisis de información.

Para generar indicadores espaciales de la situación actual hídrica, se calcula el balance hídrico de la cuenca y del sistema lacustre de estudio (Disponibilidad de agua y sistemas de recarga de la laguna), mediante la siguiente ecuación:

Ecuación Fundamental de la Hidrología

$$
\int_{d}^{i} I-\int_{d}^{i} O=\frac{\Delta S}{\Delta t}
$$

Fuente: Monsalves, 2009

Donde i es ingresos de agua a la microcuenca, I: precipitación P, Importaciones de agua, escorrentía superficial desde otras hoyas, aguas subterráneas desde otras hoyas y las salidas de agua de la microcuenca son 0: correspondiendo a evaporación, transpiración, escorrentía superficial hacia otras hoyas, exportaciones de agua, aguas subterráneas hacia otras hoyas, infiltración que da como resultado el cambio de almacenamiento.

Con estos datos se obtienen mapas de descriptores ambientales como indicadores espaciales de la oferta y demanda hídrica (Barberian, 2014) de déficit hídrico, Isoyetas, Isotermas, que serán usados para determinar la distancia de los círculos concéntricos de análisis para el uso de los métodos de costos de viaje (USFQ, 2015), se calculan zonas hidrológicas homogéneas, y mediante programación estructurada en GIS con la herramienta phyton se establece la distancia de buffer más adecuada para la realización de círculos concéntricos superponiendo los datos antes mencionados.

Es necesario identificar instrumentos económicos que definan la capacidad que tiene un espacio natural para satisfacer necesidades (Azqueta, 2002, p. 53), se toma en cuenta que la 
laguna no solo es un lugar donde se recopila el agua, sino que tiene además de belleza escénica, influencia directa en la regulación del ciclo hídrico; es un sistema de soporte de biodiversidad (Barzev, 2002), por lo cual el servicio ambiental dependerá en gran medida de su conservación (Díaz et al., 2005).

Para valorar bienes que no tienen mercado, se lo realiza con la técnica econométrica de modelo de coste de viaje (CEPAL, 2012), minimizando el valor de incertidumbre con datos de trabajo de campo (EUMED, 2009), simulando un mercado que no existe (CEPAL, 2012).

Debido a las actividades económicas que compiten con la conservación de la laguna y que generan réditos inmediatos a la población del sitio, se consideró el costo que la población está dispuesta a pagar para llegar al sitio (Barrantes y Vega, 2001), para lo cual se usa la estimación de precio del mercado (Bateman et al., 2003), que genera una encuesta, en cuanto a preguntas de disposición de pago DAP realizada a los actores administrativos directos (Célleri, 2009; Buytaert et al., 2006; Biao et al., 2010), como son representes del MAE, GAD Riobamba, EMAPAR (Muñoz-Pina et al., 2008).

El dato de disponibilidad hídrica se lo considera como el activo y, por lo tanto, tiene un potencial económico de la zona (Odum, 1986, citado en Barrantes y Vega, 2001), que mediante el método de costos de viaje obtiene un valor monetario equivalente a su uso recreativo, a partir del valor generado para llegar al sitio (Del Saz Salazar, 1997).

La aproximación estadística se la realizó tipo individual (Tempesta y Thiene, 2006), donde el costo de ingreso es cero, y el individuo se traslada al sitio, se lo realizó bajo cuatro supuestos:

a. El visitante maximiza su utilidad, en lo referente a las restricciones de ingreso y tiempo, no existe otro lugar con características naturales similares.

b. Cada viaje se lo realiza con un solo propósito, y su costo de oportunidad es el tiempo dedicado a la recreación.

c. El tiempo es de libre elección (Miranda, 2013).

d. Se aplicó la ecuación costos de viaje, donde el costo total es igual al costo del viaje + Costo del tiempo de viaje (Font, 2000).

Para determinar el valor económico probable considera no solo una población muestra de la parroquia de Cebadas, cantón Guamote, ciudad de Riobamba, sino los probables sitios desde donde se puede visitar la laguna, tomando solamente ciudades importantes en el país.

Se requiere determinar el mayor valor monetario probable para el agua de la laguna, mismo que debe ser menor a la máxima disposición de pago (DAP) de la población encuestada (De la Fuente, L. G., y Vuelta, A. C. 2004) o su mínima disposición a ser compensado (DAC) (González, 1996) para considerar conservar el sistema.

\section{Resultados y Discusión}

Al analizar los egresos de agua de la microcuenca del río Atillo, la evapotranspiración potencial (ETP), que implica necesariamente la necesidad de agua en la cobertura vegetal e influye de manera decisiva en la economía de la zona.

Se calculó la distribución temporal para un año de la evapotranspiración potencial que oscila entre $42.13 \mathrm{~mm}$ en el mes de febrero, hasta $48.86 \mathrm{~mm}$ en el mes de octubre, no tiene períodos secos debido a que la microcuenca siempre tiene más del 50 \% de agua retenida en el suelo, es decir el porcentaje de evapotranspiración potencial siempre es menor al porcentaje de evapotranspiración real, en la Figura 1 se puede apreciar los resultados del geo-cálculo realizado. 
Figura 1. Geo-cálculo para isoyetas, isotermas, ETP y déficit hídrico de la Laguna Atillo
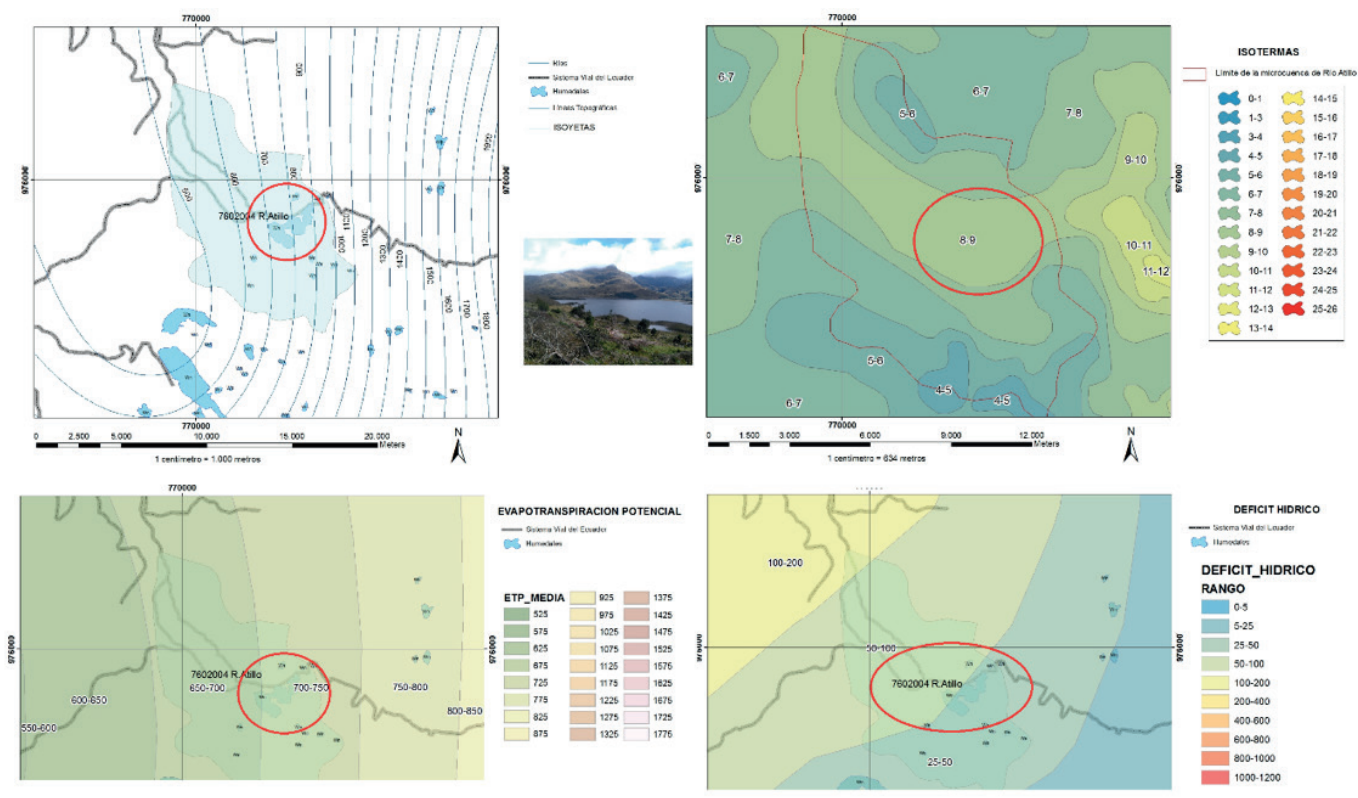

Se comparó temporalmente las imágenes disponibles, en los años 1970, 2015 para la misma fecha, esto es 30 noviembre a las 11:00, se puede observar una disminución en el espejo de agua en el área de inundación, y el cálculo de volumen corresponde al mes de noviembre, con precipitaciones medias, alto ETP, sin déficit hídrico en el mes, Figura 2.

Figura 2. Observación de diferencia y retroceso de la laguna año 1970, y 2015.

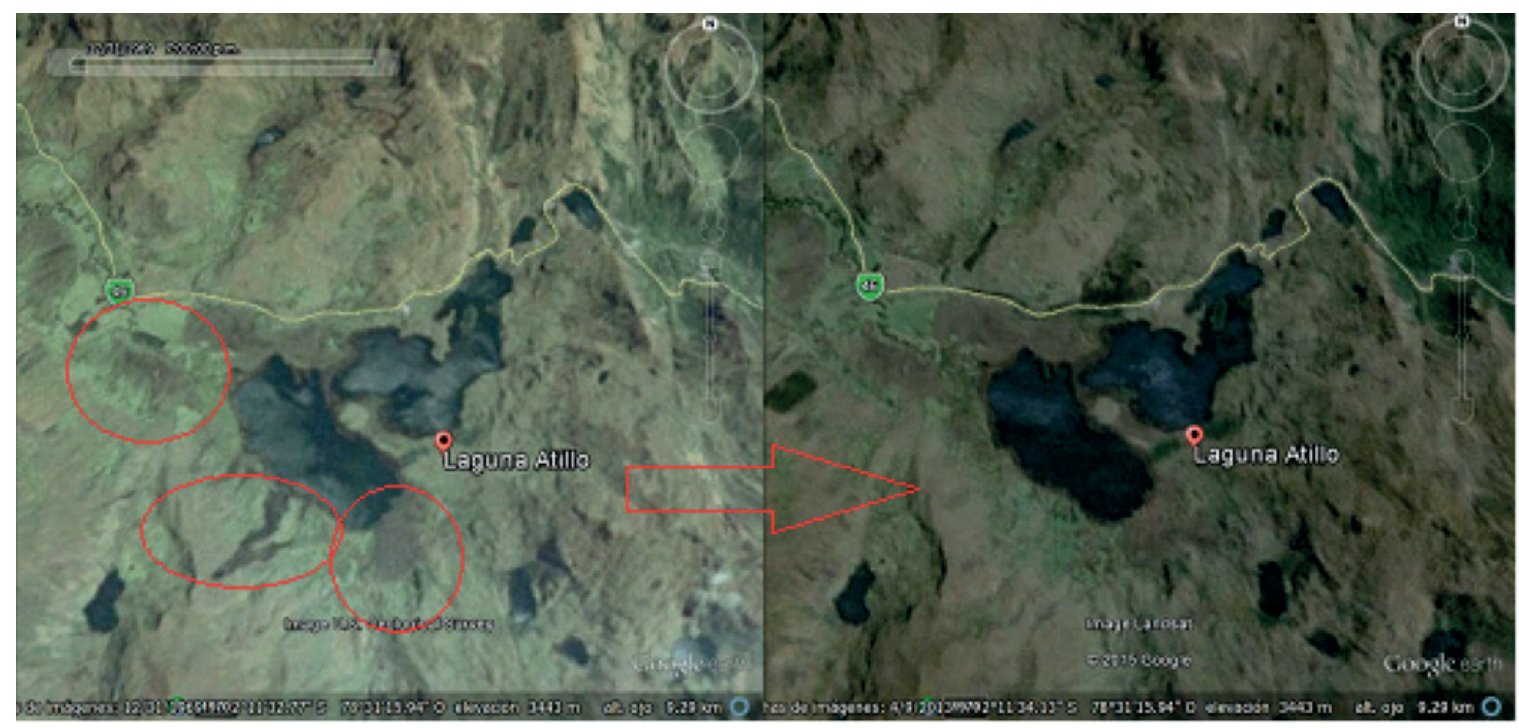

Por encontrase dentro de un parque nacional, como es el Parque Nacional Sangay, no tiene poblaciones inmediatamente cercanas, sin embargo, es considerado como centro de turismo de la zona para las poblaciones inmediatas de Riobamba, Palmira, Tixán, Multitud, Alausí, Sibambe, Guasuntos, Ventura, Pistishi, Gonzalo, Achupallas, Huigra Chuncha, Capzol, Compud, 
Llagos, General Morales, Suscal, Ducur, Zhud, Juncal, El Tambo, Ingapirca, Cañar, Choropote, Rivera, Pindilig, Amaluza, Today, Guarainag.

De acuerdo a los datos, la mayor cantidad de población relevante, se encuentra en San Pedro de Riobamba, con la mayor cantidad de visitantes al año, según la encuesta realizada a la administración de la laguna. Para obtener los datos sobre el número de visitantes al año a la laguna, se entrevistó al señor Pablo Toaza, guarda-parque del Ministerio del Ambiente de Riobamba, y el señor ing. Cristian Clavijo, responsable del Parque Nacional Sangay, del ministerio del Ambiente, así como consultas a la Dirección Nacional de Biodiversidad, que lleva un recuento de turistas nacionales y extranjeros que llegan al sitio y que coincide con el anuario estadístico 2014 del ministerio de Turismo del Ecuador, cuyo resultado se observa en la Figura 3.

Figura 3. Visitantes al sitio por mes

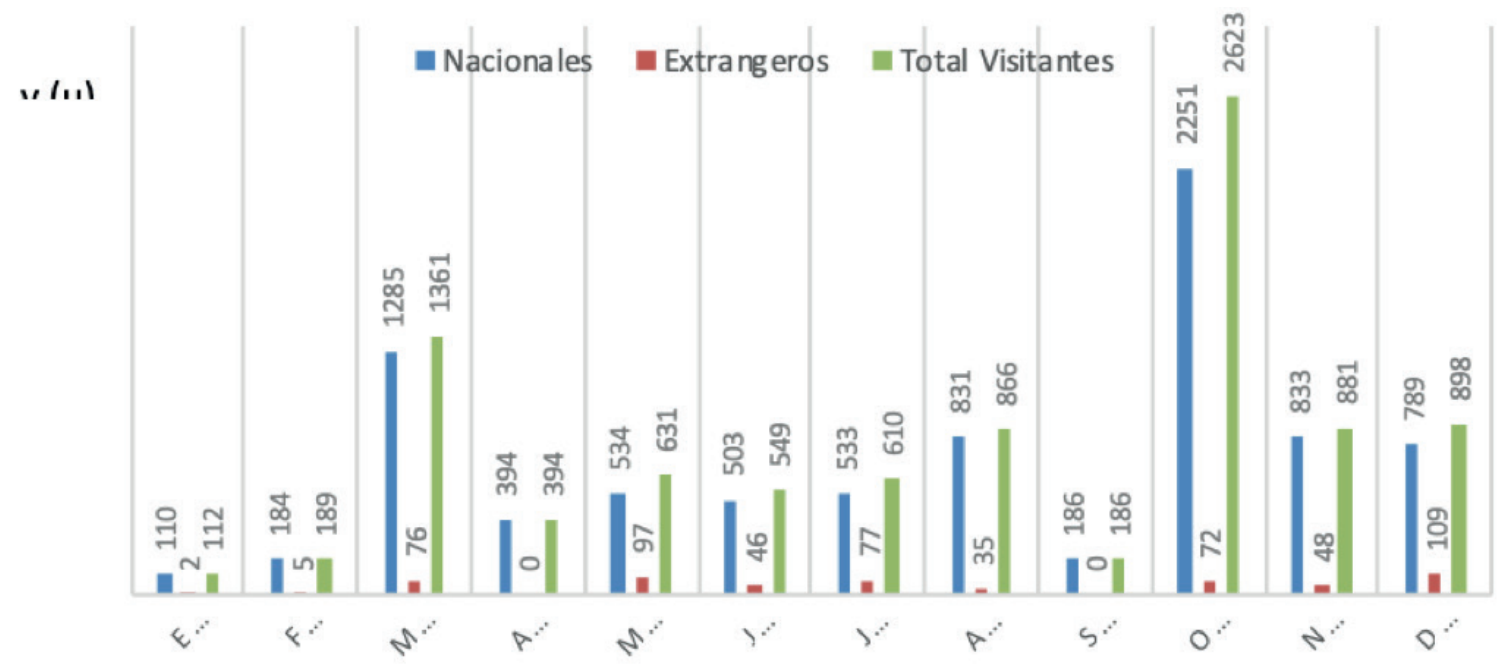

Fuente: MAE, 2014; MINITUR, 2014.

Elaborado por Mónica Delgado.

Para el cálculo de costo oportunidad se considera la estimación de precios, mediante la estimación de precio del mercado (Bateman et al., 2003), que genera una encuesta en cuanto a preguntas de disposición de pago DAP. La encuesta fue realizada exclusivamente a cuidadores del parque, autoridades involucradas y delegados del Ministerio de Turismo y Ambiente, a fin de recopilar información certera que permita realizar el cálculo (Muñoz-Pina et al., 2008).

Una vez obtenidos los datos se aplicó la ecuación Costos de viaje, donde el costo total es igual al costo del viaje + Costo del tiempo de viaje (Font, 2000), tomando como base el tiempo de permanencia promedio que no supera un día.

$$
\max _{x, z}\left\{u(x, y) \dot{u} y=c x+p z, T=h+x\left(t_{1}+t_{2}\right)\right\}
$$

De acuerdo a la definición variables (Haab y Mc Connell, 2002)

\footnotetext{
x Número de viajes

C Costos para transportarse al sitio

t tiempo para cada viaje
} 
T Tiempo total del viaje

t2 tiempo de permanencia en el espacio natural

z otros atractivos de la zona

p precio del conjunto de otros bienes

y ingreso exógeno

h tiempo trabajo

Usando la metodología de Salazar (2012) para el cálculo econométrico del coste de viaje, se ha definido mediante un buffer en ARC GIS 10.2, zonas de estudio dentro de círculos concéntricos sobre el territorio más probable de visita, de tal manera que se abarca los principales centros poblados.

Se estableció el número de visitas por zona realizadas en el último año, de esta manera eliminar lo más posible la incertidumbre (Arguello, 2007), siendo el total de visitas por zona y su relación por cada mil habitantes de cada zona (Thomas, Vidal, y Chacur, 2014), para lo cual se realizó una campaña de veinte días de recorrido por las poblaciones de Palmira, Tixán Guasuntos, Pumallacta, Sevilla, Achupallas, Cebadas, Guamote, Alausí, Simbabe, Pishishi, Gonzol, Chunchi, Coalzol, La Candela, Penipe, Valparaíso, Guano, San Gerardo, Cubies, Riobamba, Villa Unión, Calpi, Pungalá, Licto, Flores, Columbre, Huigra, Conoud, Llagos, Quimiag, Chambo, San Luis, Cacha, Punín, Santiago de Quito, Juan de Velasco, Pallatanga Multitud;siempre iniciando desde la laguna con el fin de comprobar los tiempos de llegada, y el uso de la gasolina en cada recorrido, con lo cual se valida el método de tiempos concéntricos.

El valor de viaje se lo realizó suponiendo una velocidad de $90 \mathrm{~km} / \mathrm{h}$, además tomando en cuenta la metodología de costos de viaje, la distancia se considera igual a los respectivos círculos concéntricos considerados, inclusive si el trayecto de viaje representa mayor distancia a la estimada debido a la dificultad de los caminos para llegar al sitio (UNAM, 2015). Esto se puede observar en la Figura 4.

El valor medio del costo por kilómetro se lo multiplica por la distancia ida y vuelta y se obtiene el costo de viaje para cada círculo concéntrico en relación con el número de visitas /1000. Se lo visualiza en la tabla 1.

Tabla 1. Cálculo de Costo de Viaje en relación con el número de visitas

\begin{tabular}{|c|c|c|c|}
\hline Zona & $\begin{array}{c}\text { Distancia ida y vuelta } \\
(\mathbf{k m})\end{array}$ & Costo de viaje & Visitas/100 \\
\hline 1 & 20 & 222,862 & 0.021 \\
\hline 2 & 40 & 445,724 & 0.444 \\
\hline 3 & 60 & 668,587 & 0.768 \\
\hline 4 & 80 & 891,449 & 0.625 \\
\hline 5 & 100 & 1114,31 & 0.618 \\
\hline 6 & 120 & 1337,17 & 0.609 \\
\hline 7 & 140 & 1560,04 & 0.591 \\
\hline 8 & 160 & 1782,9 & 0.528 \\
\hline 9 & 180 & 2005,76 & 0.525 \\
\hline 10 & 200 & 2228,62 & 0.429 \\
\hline 11 & 220 & 2451,48 & 0,395 \\
\hline 12 & 240 & 2674,35 & 0,392 \\
\hline
\end{tabular}




\begin{tabular}{|c|c|c|c|}
\hline 13 & 260 & 2897,21 & 0,387 \\
\hline 14 & 280 & 3120,07 & 0,385 \\
\hline 15 & 300 & 3342,93 & 0,38 \\
\hline 16 & 320 & 3565,8 & 0,359 \\
\hline 17 & 340 & 3788,66 & 0,354 \\
\hline 18 & 360 & 4011,52 & 0,347 \\
\hline $19-25$ & 500 & 5571,56 & 0,316 \\
\hline $26-40$ & 800 & 8914,49 & 0,26 \\
\hline
\end{tabular}

Elaboración: Mónica Delgado 2015.

Fuente: Trabajo de campo medios de transporte particular, transporte contratado o cooperativa de transportes Unidos y Riobamba.

Figura 4. Cálculo de las distancias en círculos concéntricos

\section{LAGUNA MAGDALENA, COMPLEJO LACUSTRE ATILLO- RIOBAMBA ECUADOR}
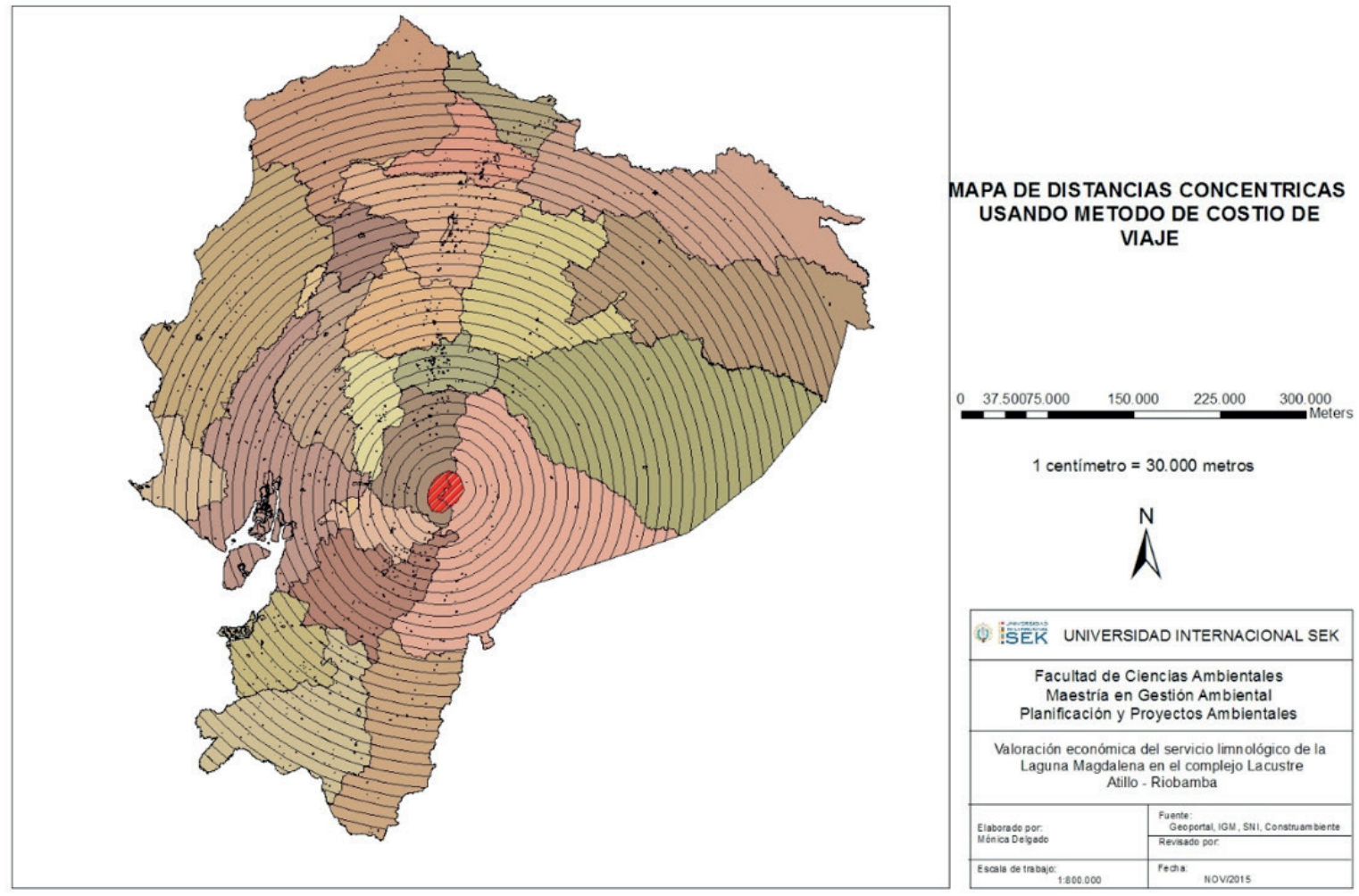

El tiempo para cada viaje por cada círculo concéntrico 1 hora: 10 minutos, por ejemplo, desde la ciudad de Riobamba hasta la laguna Magdalena, recorriendo la vía a San Luis, Flores, Cebadas, vía a Macas es de 3 horas con treinta minutos aproximadamente, mismo tiempo se realiza al tomar otro camino dentro del mismo círculo concéntrico.

Se usa la regresión de poisson para relacionar las variables consideradas para el estudio, se lo visualiza en la Figura 5. 
Figura 5. Regresión de Poisson en relación con la distancia de viaje.

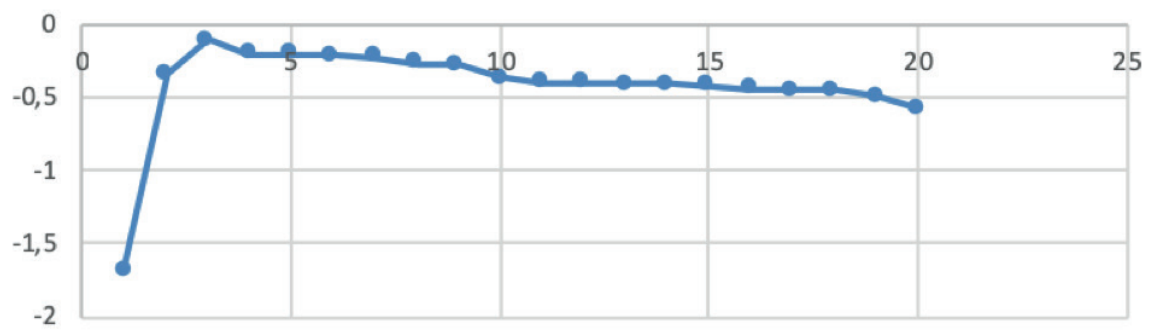

Elaborado por: Mónica Delgado.

Los beneficios de visita a la laguna se encontraron en función de la distancia en círculos equidistantes de $10 \mathrm{~km}$ cada uno, siendo el máximo beneficio obtenido a los $35 \mathrm{~km}$, y en adelante disminuye, no hay beneficios de visita desde el mismo punto, al ser un espacio no habitado.

A mayor distancia, se incrementa el costo de viaje de manera lineal hasta los $170 \mathrm{~km}$ en vía terrestre, a partir de ese momento el costo se vuelve exponencial, siendo el mayor gasto a 190 km, valor económico que crece aceleradamente hasta los 400 km de viaje. En la Figura $6 \mathrm{se}$ puede observar la distancia ida y vuelta en relación al costo de viaje, en tanto que en la Figura 7 se visualiza función demanda recreativa por zonas concéntricas equidistantes.

Figura 6. Distancia ida y vuelta en relación al costo de viaje

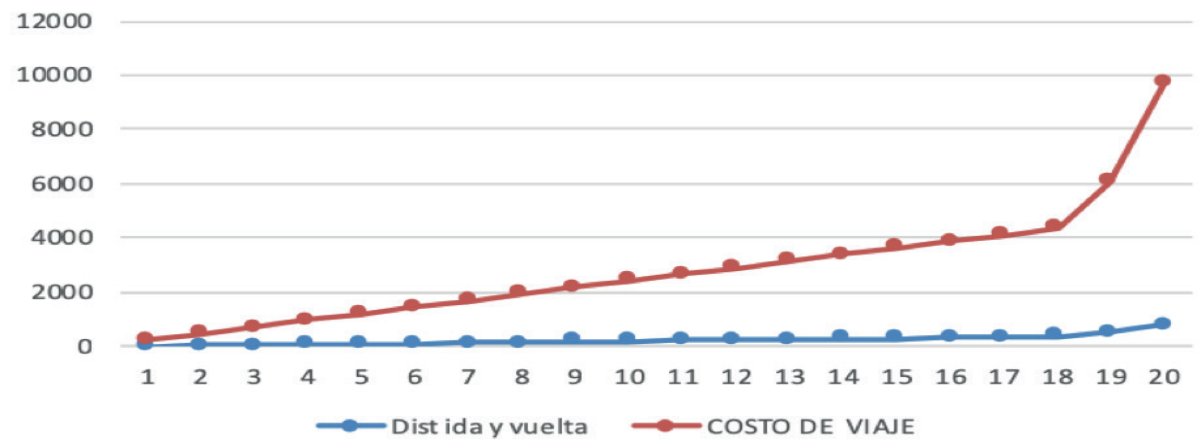

Elaborado por Mónica Delgado.

Figura 7. Visitantes al sitio por mes

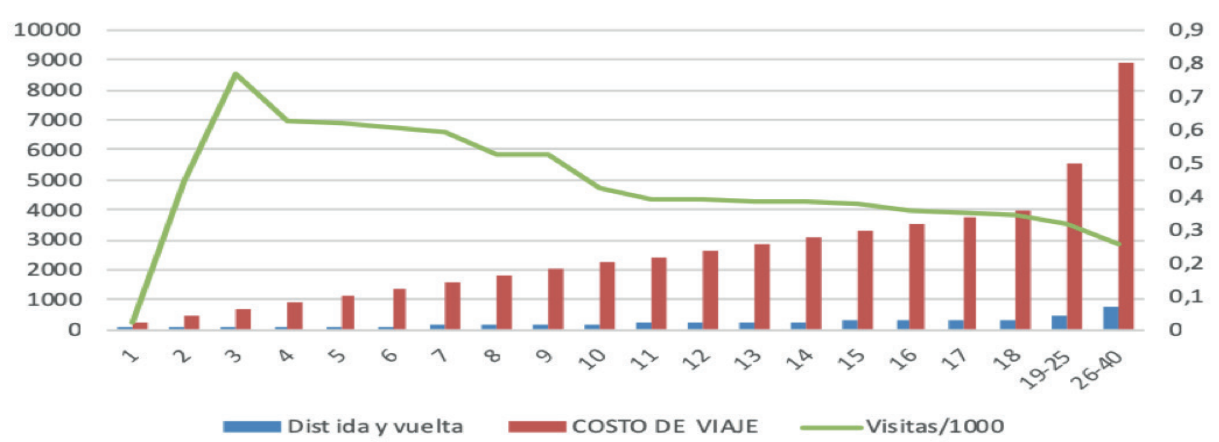

Elaborado por Mónica Delgado. 
La demanda recreativa disminuye conforme la distancia aumenta de manera no lineal

Para el cálculo del costo de tiempo se ha considerado la unidad del salario mínimo vital como unidad, ver Figura 8.

El costo del tiempo se determina basándose en funciones lineales, se puede ver qué pendiente de tiempo en minutos vs. círculos concéntricos es 4.44, en tanto que la pendiente del costo de viaje vs. círculos concéntricos es 1.54, calculado a partir de la fórmula de pendiente, $\mathrm{y} / \mathrm{x}$ igual $\mathrm{m}^{1}-\mathrm{m}^{2} / \mathrm{v} 1-\mathrm{v} 2$, con lo cual se puede concluir que para el consumidor promedio el tiempo en ir y volver desde la zona de estudio es mayor que el tiempo de permanencia en sitio, lo que contribuye a disminuir su atractivo turístico, y queda representado en la tabla 2.

Tabla 2. Cálculo de costo de tiempo para costo de viaje en relación con el número de visitas.

\begin{tabular}{|c|c|c|c|c|}
\hline Zona & $\begin{array}{c}\text { Tiempo ida y velta } \\
\text { (minutos) }\end{array}$ & $\begin{array}{c}\text { Costo por unidad } \\
\text { con base al SMV } \\
\text { (minutos) }\end{array}$ & Costo del tiempo & Visitas/1000 \\
\hline 1 & 180 & 0.075 & 13.5 & 0.021 \\
\hline 2 & 360 & 0.075 & 27 & 0.444 \\
\hline 3 & 540 & 0.075 & 54 & 0.768 \\
\hline 4 & 720 & 0.075 & 67.5 & 0.625 \\
\hline 5 & 900 & 0.075 & 81 & 0.609 \\
\hline 6 & 1080 & 0.075 & 94.5 & 0.591 \\
\hline 7 & 1260 & 0.075 & 108 & 0.528 \\
\hline 8 & 1440 & 0.075 & 121.5 & 0.525 \\
\hline 9 & 1620 & 0.075 & 148.5 & 0.429 \\
\hline 10 & 1800 & 0.075 & 162 & 0.395 \\
\hline 11 & 1980 & 0.075 & 175.5 & 0.392 \\
\hline 12 & 2160 & 0.075 & 189 & 0.387 \\
\hline 13 & 2340 & 0.075 & 202.5 & 0.385 \\
\hline 14 & 2520 & 0.075 & 216 & 0.38 \\
\hline 15 & 2700 & 0.075 & 229.5 & 0.359 \\
\hline 16 & 2880 & 0.075 & 243 & 0.354 \\
\hline 17 & 3060 & 0.075 & 337.5 & 0.347 \\
\hline 18 & 3240 & 0.075 & 540 & 0.316 \\
\hline $19-25$ & 4500 & 0.075 & & 0.26 \\
\hline $26-40$ & 7200 & 0.075 & & \\
\hline & & & & \\
\hline
\end{tabular}

Figura 8. Relaciones del costo de tiempo

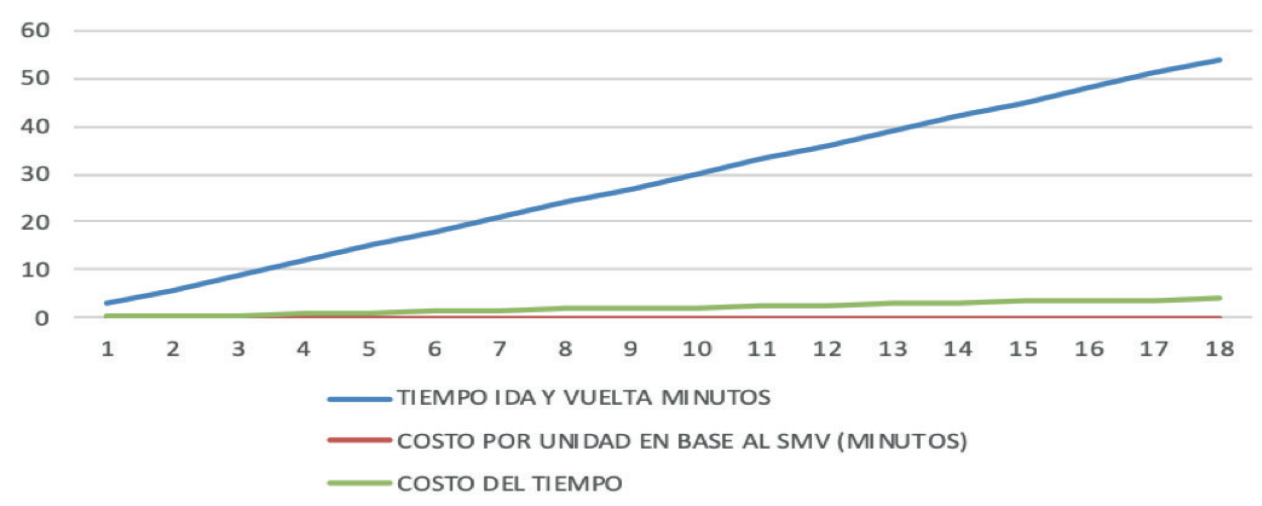

Elaborado por Mónica Delgado. 
El valor del agua en el caso más costoso es de 0.29 ctvs. por $\mathrm{m}^{3}$ por habitante, tomado de los valores en Riobamba para uso de agua potable, y el cual representa nuestro parámetro de comparación superior.

Para los 8733 visitantes a la laguna en el año 2014 el costo del consumo de agua potable, considerando que cada persona requiere 160 litros diarios (ETAPA, 2010), es de $0.29 \mathrm{ctvs}$. el $\mathrm{m}^{3}$ (EMAPAR, 2015), el costo del agua almacenada en la laguna sin considerar su potencial como agua subterránea es de USD 405.21 por recarga, y el costo de viaje para personas entre 15 y 65 años, es de USD 1.05 promedio por lo que el costo del almacenamiento para fines de conservación y turismo es de USD 8967.59, en las tablas 3 y 4 se puede ver el cálculo de costo por tiempo de viaje en relación con el número de visitas.

Tabla 3. Cálculo de costo de tiempo para costo de viaje en relación con el número de visitas

\begin{tabular}{|l|c|c|}
\hline $\begin{array}{c}\text { Costo del agua potable } \\
\mathrm{m}^{3} \text { /por habitante (centavos) }\end{array}$ & $\begin{array}{c}\text { Visitantes al año } \\
\text { (personas entre 15 años y 65) }\end{array}$ & $\begin{array}{c}\text { Volumen de agua consumida } \\
\text { por habitante (I) }\end{array}$ \\
\hline 0.29 & 8733 & 160 \\
\hline Costo total del agua en $\mathrm{m}^{3}$ : & & USD 405.21 \\
\hline
\end{tabular}

* $1 \mathrm{~m}^{3}$ igual a 1000 litros.

Fuente: EMAPAR. Elaborado por Mónica Delgado.

Nota 1: La elaboración se refiere a datos de la evaluación financiera para un extracto de proyección realizada desde 2011 hasta el año 2050 por EMAPAR.

Tabla 4. Costo del tiempo de viaje

\begin{tabular}{|l|c|c|}
\hline $\begin{array}{c}\text { Visitantes al año } \\
\text { (personas entre 15 años y 65) }\end{array}$ & $\begin{array}{c}\text { Costo de distancia } \\
\text { de viaje (USD) }\end{array}$ & $\begin{array}{c}\text { Costo del tiempo de viaje } \\
\text { (USD) en la distancia } \\
\text { más productiva }\end{array}$ \\
\hline 8733 & 8914.50 & 53.1 \\
\hline Costo total del agua en $m^{3}$ : & & USD 8967.59 \\
\hline
\end{tabular}

Fuente: EMAPAR. Trabajo de Campo. Elaboración. Mónica Delgado.

El uso recreacional del lugar está alrededor de USD 8967.59 es decir USD 1 por visita, en tanto que el uso del agua en el caso supuesto de darle un uso directo de consumo es de USD 405.21 que equivale a 0.05 centavos por visita.

\section{Conclusiones y recomendaciones}

Los resultados mencionados han permitido cumplir los objetivos; la laguna Magdalena, ubicada en la provincia de Chimborazo, cantón Alausí, parroquia Achupallas, en la frontera provincial que limita con la ciudad de Macas, no ha sido explotada en su potencial turístico. Se comprueba también la dificultad de acceso al lugar y a la estadía para pernoctación por lo que el tiempo de permanencia no supera el día, por tanto se concluye que se cumplieron los objetivos.

La caracterización pluviométrica se la obtuvo con el análisis de la variabilidad mensual o distribución intra-anual (régimen de precipitación), se obtienen dos estaciones definidas: una por un período de abundantes lluvias comprendido entre enero y abril, y el segundo período con menor precipitación y que comprende los meses de mayo, junio, julio, agosto, noviembre y 
diciembre, sin embargo, se tiene un pico lluvioso menor en los meses de septiembre, octubre, con valores que varían de $500 \mathrm{~mm}$ a $1100 \mathrm{~mm}$.

El parámetro temperatura es el de mayor importancia para el análisis económico, debido a que se su variación afecta de manera directa la disponibilidad hídrica, tanto en la precipitación como en la humedad edáfica. Se calcularon las temperaturas medias mensuales y anuales, las que varían desde los $7.6^{\circ} \mathrm{C}$ a $8.2^{\circ} \mathrm{C}$, con un promedio anual $8{ }^{\circ} \mathrm{C}$.

Se calcula la evapotranspiración potencial (ETP), lo cual implica necesariamente la necesidad de agua para la conservación del sistema, lo que influye de manera decisiva en la economía de la zona de origen turístico, la ETP media mensual oscila entre $42.13 \mathrm{~mm}$ en el mes de febrero, hasta $48.86 \mathrm{~mm}$ en el mes de octubre.

En ningún caso hay secado de la laguna, hecho que la convierte en un atractivo turístico permanente, con un alto grado de fragilidad que depende también del sostenimiento de la vegetación circundante.

Entre la población relevante, se encuentra San Pedro de Riobamba, con la mayor cantidad de visitantes al año, esta ciudad tiene una extensión aproximada de $30 \mathrm{~km}^{2}$, donde la población urbana es de 124807 (INEC, 2001) cuenta con 38855 predios urbanos registrados, seguida de las comunidades de Palmira, Tixán, Multitud, Alausí, Sibambe, Guasuntos, Ventura, Pistishi, Gonzalo, Achupallas, Huigra Chuncha, Capzol, Compud, Llagos, General Morales, Suscal, Ducur, Zhud, Juncal, El Tambo, Ingapirca, Cañar, Choropote, Rivera, Pindilig, Amaluza, Today, Guarainag.

El total de visitantes año registrados para el 2014 es 8733, siendo los meses de marzo y octubre los más visitados, suponiendo una velocidad de $90 \mathrm{~km} / \mathrm{h}$, el costo promedio de gasolina por kilómetro es de USD 11.14, con un costo de gasolina extra de USD 1.48 y litro de gasolina a 40 centavos de dólar.

El valor medio del costo por kilómetro se lo multiplica por la distancia ida y vuelta y se obtiene el costo de viaje para cada círculo concéntrico en relación con el número de visitas /1000, obteniéndose el valor de <t> tiempo para cada viaje por cada círculo concéntrico1 hora 10 minutos.

A mayor distancia, se incrementa el costo de viaje de manera lineal hasta los $170 \mathrm{~km}$ por vía terrestre; a partir de ese momento el costo se vuelve exponencial, siendo el mayor gasto a $190 \mathrm{~km}$, valor económico que crece aceleradamente hasta los $400 \mathrm{~km}$ de viaje terrestre.

El uso recreacional del lugar está alrededor de USD 8967.59 es decir USD 1 por visita, en tanto que el uso del agua en el supuesto caso de darle un uso directo de consumo es de USD 405.21 que equivale a 0.05 centavos de dólar por visita.

El excedente del consumidor para método del costo de viaje, nos da como resultado que el valor del excedente es positivo, incluso sin cobro de entrada a la laguna, por lo cual se concluye que vale la pena conservar la riqueza hídrica de la laguna.

\section{Recomendaciones}

Se debe impulsar el turismo científico a la zona, e incrementar acciones desde la municipalidad de Riobamba para mejorar el turismo en general, a base de sus atractivos: 324 lagunas, entre las más importantes la laguna de Ozogoche, las de Atillo, entre las que están la laguna Magdalena o laguna Negra, laguna de Colta en Balbanera, así como algunas montañas y nevados; entre los que destacan El Chimborazo (6310 m.s.n.m.), el Altar (5319 m.s.n.m.), Carihuairazo (5116 
m.s.n.m.), volcán Tungurahua (5033 m.s.n.m.), el Parque Nacional Sangay y los viajes en tren (EMAPAR, 2015).

Se debe incrementar acciones para la conservación de la riqueza hídrica, y se recomienda realizar un estudio de disminución de volumen de la laguna, así como efectos del cambio climático global, por encontrar variación en las fotos satelitales.

\section{Bibliografía}

AEMTE (2014). Anuario estadístico 2014 del ministerio de Turismo del Ecuador, rescatado el 30 de noviembre de 2015 de http://www.turismo.gob.ec/

Aguilera Klink y Vicent Alcántara (1990) De la economía ambiental a la economía ecológica Federico CIPEco-social (fuhem.es/cip-ecosocial/)C/ Duque de Sesto 40, pp. 56-75

Álvarez, R., Fernández, H., y Rua, J. (2009). Matemáticas básicas con aplicaciones a las Ciencias Económicas y afines. (2. ed.). Bogotá, Colombia: Universidad de Blasco.

Azqueta, D. (1999). "Ventajas Comparativas y explotación de los recursos naturales". En: Revista de la CEPAL. 68, 115-134, CEPAL, 2012 manual de evaluación de contingente rescatado el 19 de diciembre de 2015 de http://www.cepal.org/ilpes/noticias/paginas/0/35060/manual_evaluacion_contingente.pdf

Azqueta, Diego (2002), Introducción a la Economía Ambiental España: Mc craw-Hill/Interamericana de España. pp. 53.

Babier, 1989 Métodos de valoración económica para ecosistemas naturales no intervenidos rescatada el 15 de diciembre de 2015 de Barberán, Albert; Henley, Jessica; Fierer, Noah; Casamayor, Emilio 0. Structure, inter-annual recurrence, and global-scale connectivity of airborne microbial communities. Science of the Total Environment 487, 187-195 (2014) DIGITAL.CSIC

Barzev, R. (2002). Guía metodológica de valoración económica de bienes, servicios e impactos ambientales. Proyecto para la consolidación del corredor biológico mesoamericano. Serie técnica, 4, 149.

Buchanan, J. T., Henig, E. J., y Henig, M. I. (1998). Objectivity and subjectivity in the decision making process. Annals of Operations Research, 80, 333-345. Buenos Aires: Siglo XXI Editores.

Cabeza, Á. M. (2002). Ordenación del territorio en América Latina. Scripta Nova: Revista electrónica de geografía y ciencias sociales, 6, 125.

Camacho, D. C. (2008). Esquemas de pagos por servicios ambientales para la conservación de cuencas hidrográficas en el Ecuador. Investigación agraria. Sistemas y recursos forestales, 17(1), 54-66.

Campos, M. (2010), Economía Verde, Editorial Cegesti, 151, 1-4.

Carbal Herrera, A. (2009). La valoración económica de bienes y servicios ambientales como herramienta estrategica para la conservación y uso sostenible de los ecosistemas: "Caso Ciénaga La Caimanera, Coveñas-Sucre, Colombia" Criterio Libre.

Castro, M. (2011). Proyecto Creación de Capacidades para Valoración Socio-económica de los humedales altoandinos. La experiencia en Oña-Saraguro-Yacuambi y el frente sur occidental de Tungurahua.

CEPAL. (2012). COSTOS DE VIAJE, http://herzog.economia.unam.mx/profesores/blopez/valoracion-travel.pdf

Chang, M. Y. (2005). La economía ambiental. Sustentabilidad, 165-178. Tesis de Maestría. FLACSO Ecuador.

Checkland, P., y Scholes, J. (1990). Soft System Methodology in Action. Wiley,

Chichester. de la Fuente, L. G., y Vuelta, A. C. (2004). Métodos directos e indirectos en la valoración económica de bienes ambientales. Aplicación al valor de uso recreativo del Parque Natural de Somiedo. Estudios de economía aplicada,22(3), 811-838.

Chiluiza, L. (2001). Gobierno municipal del cantón Santiago de Píllaro. Gobierno parroquial autónomo descentralizado rural de Emilio María Terán. Plan de desarrollo y ordenamiento territorial parroquia Emilio María Terán. 
Cruz, G. (2005). Economía aplicada a la valoración de impactos ambientales. Madrid. España: Universidad de Caldas.

De la Fuente, L. G., y Vuelta, A. C. (2004). Métodos directos e indirectos en la valoración económica de bienes ambientales. Aplicación al valor de uso recreativo del Parque Natural de Somiedo. Estudios de economía aplicada, 22(3), 811-838.

Declaración Universal de la UNESCO sobre la Diversidad Cultural, octubre de 2004. Consultado el 29 de abril de 2013.Leff, Enrique (2001), Racionalidad ambiental, democracia participativa y desarrollo sustentable.

Del Saz Salazar, S. (1997). Los métodos indirectos del coste de viaje y de los precios hedónicos: Una aproximación. Revista española de economía agraria, (179), 167-190.

DRHGA. Dirección de recursos hídricos y gestión ambiental. (2013). Boletín meteorológico trimestral de Tungurahua.

Ecologíahoy. (2012). Linmología, http://www.ecologiahoy.com/limnologia

Encalada, G. (2006). Pago por servicios ambientales del recurso hídrico como

EUMED. (2009) Valor de opción. http://www.eumed.net/libros-gratis/2009

FAO. (2003). Resumen Ejecutivo. Foro Regional Sistemas de Pago por Servicios Ambientales en Cuencas Hidrográficas. 9 al 12 de Junio 2003. INRENA-REDLACH-FAO, Arequipa, Perú. URL: http://www. rlc.fao.org/prior/recnat/foro/resumen.pdf (Consulta: 5 mayo, 2007).

FAO (2014). Valoración de os bosques, contexto, problemas y directrices rescatado el 12 de enero de 2016 http://www.fao.org/docrep/008/v7395s/v7395s07.htm\#TopOfPage

Figueroa, J. R. (2005). Valoración de la biodiversidad: Perspectiva de la economía ambiental y la economia ecológica. Interciencia, 30(2), 103-107. Recuperado en 21 de enero de 2016, de http://www.scielo. org.ve/scielo.php?script=sci_arttext\&pid=S0378-18442005000200011\&lng=es\&tlng=es

Font, A. R. (2000). Valoración económica de los atributos ambientales mediante el método del coste de viaje. Estudios de economía aplicada, (14), 173-198.

Glover, D. (2010). Valorizar el medio ambiente: Economía para un futuro sostenible. España. IDRC, 59-60.

González, C. J. L. (1996). Valoración contingente de espacios naturales en Gran Canaria: El valor de no-uso y el efecto del formato. En Gestión de espacios naturales: la demanda de servicios recreativos, 125-142. Madrid, España: McGraw-Hill Interamericana de España.

Hofstede, R., y Mena, P. (2000). Beneficios escondidos del páramo: servicios ecológicos e impacto humano. En: Foro electrónico: los páramos como fuente de agua: mitos, realidades, retos y acciones. URL: http://www.infoandina. org/apc-aa-files/237543fdce333f3a56026e59e60adf7b/ II_conferencia_paramos.pdf (Consulta: 1 nov, 2014)

INEC, UNFPA y AME. Instituto Nacional de Estadísticas y Censos, Fondo de población de las Naciones Unidas. (2008). Descripción del Cantón Píllaro. Distribución de población y tasas de analfabetismo.

Jiménez, M., María, J., Aguarón Joven, J., y Escobar Urmeneta, M. T. (2001). Metodología científica en valoración y selección ambiental. Pesquisa Operacional, 21(1), 1-16.

Josse, C., Mena, P. y Medina, G. (1999). El páramo como fuente de recursos hídricos. Quito, Ecuador: Abya-Yala.

Leopold. (1949). Evaluación de impacto ambiental y desarrollo económico, http://www.iadsargentina. org/?gclid=CPrhhtys-cECFTMQ7AodvloA-A

Linares, P. y Romero, C. (2012). Economía y medio ambiente: herramientas de valoración ambiental. Madrid, España: Universidad pontificia Comillas de Madrid. http://www.iit.upcomillas.es/pedrol/ documents/becker08.pdf

Lumbreras, E. L. (2002). Estrategia de conservación de flora silvestre en la Comunidad Valenciana. Conservación vegetal, (7), 12-13.

Malte, (2008). How to be a Ecological Economist, Elsevier, 66(1).

Mantilla, E. (2007). La contabilidad ambiental en el desarrollo sostenible. Revista Internacional de Contabilidad \& Auditoría, 25. 
Martínez, M., y Dimas, L. (2007). Valoración económica de los servicios hidrológicos: subcuenca del río Teculután-Guatemala. Programa de Comunicaciones WWF. Centroamérica.

Mearns L., Giorgi F., Whetton P., Pabon D., Hulme M., y Lal M., (2003). Lineaments jurídicos 2014.

Mirasgedis, S. y Diakoulaki, D. (1997). Multicriteria Analysis vs. Externalities Assessment for the Comparative Evaluation of Electricity Generation Systems. European Journal of Operational Research, 102, 364-379.

Molina Bravo, E., Serrano Zambrano, E., y Vásconez Gavilanes, J. (2009). Metodología para la valoración económica ambiental del valor existencia de la reserva ecológica manglares churute.

Montoya, J., Amusquivar, J., Flores, A., Mollo, A., y Sánchez, P. (2002). Efectos ambientales y socioeconómicos por el derrame de petróleo en el río Desaguadero. España. Fundación PIEB, 26-31.

Moreno, J. M., Santamaría, R. y Serrano, C. (1994). Selección de proyectos de inversión pública aislados con impacto ambiental. Documento de trabajo.

ONU. (2000). Programa de las Naciones Unidas para el Medio Ambiente Oficina Regional para América Latina y el Caribe: (2010). Elementos de carácter general que pueden ser utilizados por los Ministros y Jefes de Delegación para el Intercambio sobre Economía Verde.

Oyarzún, C., Nahuelhual, L., y Núñez, D. (2005). Los servicios ecosistémicos del bosque templado lluvioso: producción de agua y su valoración económica.Ambiente y Desarrollo, 20(3), 88-95.

Pacheco, J (2003). Valoración de la calidad Ambiental. CEPAL-ILPES, 1-20.

Paredes, Á. (2002). Declaratoria de Impacto Ambiental de la vía Ozogoche-El Atillo. Tesis de Magíster en Gestión Ambiental. Universidad Nacional de Chimborazo.

Paspuel, V. (2009). Valoración económica del servicio ambiental hídrico: estudio de caso del abastecimiento de agua de la ciudad de Tulcán. Tesis de Maestría en economía con mención en economía ecológica. FLACSO Ecuador.

Pearce, D. (2002). An Intellectual History Of Environmental Economics, Annual Review of Energy and the Environment, 27, 57-81.

Pearce, David W. y Turner, R. Kerry (1993), Economía de los Recursos Naturales y del Medio Ambiente, Madrid: Celeste Ediciones.

Porras I. (2003). Valorando los servicios ambientales de protección de cuencas: consideraciones metodológicas. En Foro Regional Sistemas de Pago por Servicios Ambientales en Cuencas Hidrográficas, 9 al 12 de junio de 2003. INRENA-REDLACH-FAO, Arequipa, Perú, rescatado el 2 de noviembre de 2015.

Prada, A., y Vázquez, M. (2001). Economía ambiental e sociedades. España. Consello da Cultura Galega, 135-138.

Pulido, A. (1998). Economía aplicada. Regularidades empíricas y hechos diferenciados. Anales de Economía Aplicada.

Randall A., y Farmer, M. (1995). Benefits, Costs and the Safe Minimum Standard of conservation. En The handboook of environmental economics. Capítulo 2.

Riechmann, J. y Fernández, F. (1994). Principios de Economía Ambiental, 101-111. CDMX, México: Pearson. Riera, P. (2005). Manual de economía ambiental y de los recursos naturales. Editorial Paraninfo, 118-121.

Robert, N. Stavins (2008). The New Palgrave Dictionary of Economics, 2. ed. Londres: Palgrave.

Salazar, F. (2012). Método de Costos de viaje para áreas naturales y protegidas, rescatado el 16 de enero de 2016. http://www.fsalazar.bizland.com/html/VIAJE.htm

Senplades, Secretaría Nacional de Planificación y Desarrollo. (2014). Ficha de cifras generales de Cantón Santiago de Píllaro.

Thomas, M. G., Vidal, R. A., y Chacur, A. A. (2014). Evaluación socioeconómica de proyectos con el método de Opciones Reales. Revista Ingeniería Industrial, 7(2).

Tomasini, D. (2007). Valoración económica del ambiente. Departamento de Economía, Desarrollo y Planeamiento. Facultad de Agronomía. Universidad de Buenos Aires. 
Tomasini, D. (2000). «Valoración económica del ambiente», Departamento de Economía Desarrollo y Planeamiento Agrícola Facultad de Agronomía de la Universidad de Buenos Aires, 1-16.

Wunder, S. (2006). Pagos por servicios ambientales: principios básicos esenciales. CIFOR Occasional Paper, 42(s), 1-24. 